\title{
Ampliando o Aprendizado na TV Digital com MCD-TV e Ginga
}

\author{
Marcos Vinicius de Andrade Lima, PPgCC-UERN/UFERSA, \\ marcos.engsoft@gmail.com \\ Carla Katarina Monteiro Marques, PPgCC-UERN, carlakatarina@uern.br \\ Rommel Wladimir de Lima, PPgCC-UERN, rommelwladimir@uern.br \\ José Osvaldo Mesquita Chaves, PPgCC-UERN/UFERSA, \\ oswaldo.mesquita@gmail.com \\ Thiago Reis da Silva, PPgSC-UFRN, trsilva.si@gmail.com \\ Karl Hansimuller Alelaf Ferreira, PPgCC-UERN/UFERSA, \\ karlhansimuller@gmail.com
}

\begin{abstract}
Resumo - Embora o ensino pela TV analógica seja utilizado há anos como forma de levar formação a diversas pessoas, ele apresenta alguns problemas: inexistência de interatividade; pouca clareza sobre qual nível de aprendizado o aluno deve alcançar em cada conteúdo trabalhado; e dificuldade em relacionar os conteúdos apresentados com a programação oferecida pelas emissoras de TV. Como forma de suprimir estes problemas, este trabalho apresenta MCD-TV, uma aplicação para TVDi que faz uso da interatividade disponibilizada pelo Ginga para ampliar as possibilidades de aprendizado mediado por meio da TV, trazendo uma nova experiência educativa para professores e alunos por meio da apresentação de conteúdos de forma mais significativa com a utilização de Mapas de Conteúdos e Dependências.
\end{abstract}

Palavras-chave: t-learning, oba-mc, mapa de conteúdos, mapa de dependências.

\section{Broadening Learning on Digital TV with MCD-TV and Ginga}

\begin{abstract}
Although the teaching by analog TV being used for years as a way to bring training to several people, it has some problems: inexistence of interactivity; little clarity about which level of learning the student must achieve in each content worked, and difficulties in relating the contents presented with the programming offered by TV stations. As a way to suppress these problems, this paper presents $M C D-T V$, an application for iTV that makes use of learning object OBA-MC and interactivity provided by Ginga to enlarge the possibilities of learning mediated through TV, bringing a new experience education for teachers and students through presentation of content more significantly with the use of Maps of Contents and Dependence.
\end{abstract}

Keywords: t-learning, oba-mc, contents map, dependence map.

\section{Introdução}

Desde a implantação da TV analógica no Brasil na década de 50, algumas iniciativas foram realizadas a fim de aproveitarem as potencialidades da TV para educação, principalmente por que a TV é um excelente meio de comunicação audiovisual, ocupa 
posição de destaque nos lares brasileiros, rompe fronteiras e alcança as regiões mais remotas do país (IBGE, 2011). Entre as principais iniciativas tem-se a criação de emissoras de TVs educativas e a produção de diversos programas educativos e culturais (Blois, 1996).

Entre os programas educativos produzidos pode-se citar o programa preparatório para o Exame Nacional do Ensino Médio (ENEM) do Projeto Quimeia. Este projeto produz vídeo-aulas que são transmitidas pela TV Mossoró a milhares de pessoas, abordando os diversos conteúdos do ENEM. O projeto ainda disponibiliza material de apoio por meio de revista impressa (contendo os conteúdos abordados e DVD com as vídeo-aulas), além de manter um fórum online para o esclarecimento de dúvidas dos alunos (Quimeia, 2013).

Para que um telespectador (aluno) possa participar do curso, neste modelo de Educação a Distância (EAD) pela TV, é preciso tão somente assistir aos programas/aulas (conteúdos) transmitidos pela emissora de TV e realizar exercícios contidos em vários fascículos de apoio (recursos/atividades) que são distribuídos em pontos de vendas. Qualquer tipo de contato com a organização geralmente é realizado pela Internet ou por telefone. Outras iniciativas, como o programa Telecurso da Fundação Roberto Marinho (transmitido pela Rede Globo de Televisão) e tele aulas (transmitidas por emissoras educativas), também fazem uso deste mesmo formato.

Embora este modelo seja amplamente utilizado, ele pode trazer certos problemas para o aprendizado de alguns alunos, tais como: (i) inexistência de uma definição clara entre o relacionamento dos conteúdos apresentados, principalmente por que os conteúdos estão espalhados em vários programas/aulas e fascículos; (ii) dificuldade para o aluno saber exatamente qual nível o professor está esperando que ele atinja em cada um dos conteúdos trabalhados; (iii) o aluno geralmente fica limitado ao programa/aula oferecido por uma única emissora; (iv) não existe nenhum mecanismo capaz de recomendar automaticamente programação extra (oferecida por qualquer emissora) que tenha relação com os conteúdos abordados, restringindo, portanto, as possibilidades de aprendizado do aluno.

É importante observar que os problemas elencados no modelo de programa educativo ocorrem principalmente por culpa da tecnologia utilizada: a TV analógica. Este tipo de tecnologia não permite que aja uma maior interação entre o telespectador e a emissora e/ou programa por meio do próprio aparelho de TV. A pouca interação existente limita-se a ligar/desligar a TV, mudar de canal e aumentar/baixar a intensidade do volume do áudio.

Como forma de contornar as limitações advindas da TV analógica e tornar o sistema de TV aberta mais moderno, foi publicado em 29 de junho de 2006 o Decreto ${ }^{\circ}$ 5.820. Nele estão definidas as diretrizes de implantação do Sistema Brasileiro de Televisão Digital Terrestre (SBTVD-T) na plataforma de transmissão e retransmissão de sinais de radiodifusão de sons e imagens, tendo três possibilidades advindas com a adoção do sistema: (i) transmissão digital em alta definição (HDTV) e em definição padrão (SDTV); (ii) transmissão digital simultânea para recepção fixa, móvel e portátil; e (iii) interatividade (Casa Civil, 2006).

Em 02 de dezembro de 2007 se iniciaram as transmissões do SBTVD-T, começando pela cidade de São Paulo. O sistema trouxe imagens em alta definição, som digital, guia eletrônico de programação (EPG) na própria tela da TV e a possibilidade de interação do público com a emissora e/ou programa por meio de aplicações interativas, a chamada Televisão Digital Interativa (TVDi) (DTV, 2012). 
Com toda a evolução e mudanças ocorridas no cenário da TV faz-se necessário, portanto, explorar de forma mais intensa os novos recursos e características da TVDi para uma completa solução dos problemas apresentados em programas educativos na TV analógica. Neste contexto, este artigo tem como objetivo apresentar a aplicação para TVDi Mapas de Conteúdos e Dependências na TV (MCD-TV), que faz uso do Objeto de Aprendizagem Mapa de Conteúdos (OBA-MC) definido por Silva (2013) a fim de tornar os conteúdos de cursos ou disciplinas mais significativos para professores e alunos. Além de ampliar a experiência interativa do telespectador por meio da incorporação de serviço de recomendação de programas televisivos para apoio aos conteúdos apresentados.

Para melhor entendimento, o texto está organizado da seguinte maneira: na Seção 2 é apresentado material e métodos. A Seção 3 mostra a aplicação interativa MCD-TV. E por fim, na Seção 4, são descritas as considerações finais e trabalhos futuros.

\section{Material e Métodos}

Nesta seção são descritos as principais ferramentas e métodos utilizados que possibilitam que o aprendizado de alunos pela TV Digital seja ampliado por meio da utilização da aplicação MCD-TV.

\subsection{Mapas de Conteúdos e Mapas de Dependência}

Mapa de Conteúdos (MC) e Mapa de Dependências (MD) são duas ferramentas propostas na tese de Lima (2009) que adotam uma metodologia de planejamento baseada em Objetivos Educacionais (OE), por intermédio de teorias pedagógicas consolidadas, como Aprendizagem Significativa e Taxionomia de Bloom, podendo ser utilizadas na modalidade de ensino presencial ou em EAD.

\subsubsection{Mapas de Conteúdos}

Com origem nos Mapas Conceituais, MC é uma ferramenta que busca fortalecer o processo de ensino-aprendizagem por meio do provimento de um conteúdo mais significativo para professores e alunos (Lima, 2009).

O MC possibilita a exibição de um curso/disciplina de forma gráfica, onde são apresentados seus conteúdos e as relações existentes. Com o MC o aluno consegue perceber, de forma mais clara, que é preciso, por exemplo, compreender conteúdos mais simples antes de partir para conteúdos mais complexos. Esta facilidade de percepção é rapidamente alcançada por que as relações entre conceitos no MC são hierárquicas, ou seja, conteúdos introdutórios sempre estão posicionados no topo do gráfico, enquanto os conteúdos mais complexos dirigem-se para sua base.

Na Figura 1 é possível observar um exemplo de um MC representando o primeiro nível de visão de um conteúdo programático. Os conteúdos que formam o MC podem ser simples (representado por elipse simples: “Teorias Aplicadas a EAD” e “Criação e Desenvolvimento EAD”) ou com subnível (representado por elipse composta: “Conceitos Básicos”). Cada conteúdo, no entanto, pode ter OE (representado por elipse com tonalidade mais clara: "Teorias Aplicadas a EAD" e "Criação e Desenvolvimento EAD”) e/ou recursos pedagógicos (representados por um círculo com a letra "R" anexado à elipse: todos os conteúdos do exemplo apresentado).

A ferramenta para criar o MC foi desenvolvida para Modular Object-Oriented Dynamic Learning Environment (Moodle), que é um Ambiente Virtual de 
Aprendizagem (AVA), gratuito e amplamente difundido na Internet. O funcionamento da ferramenta é simples, onde por meio de um conjunto de interações o professor é guiado na elaboração do planejamento de um curso/disciplina e no final o MC é gerado.

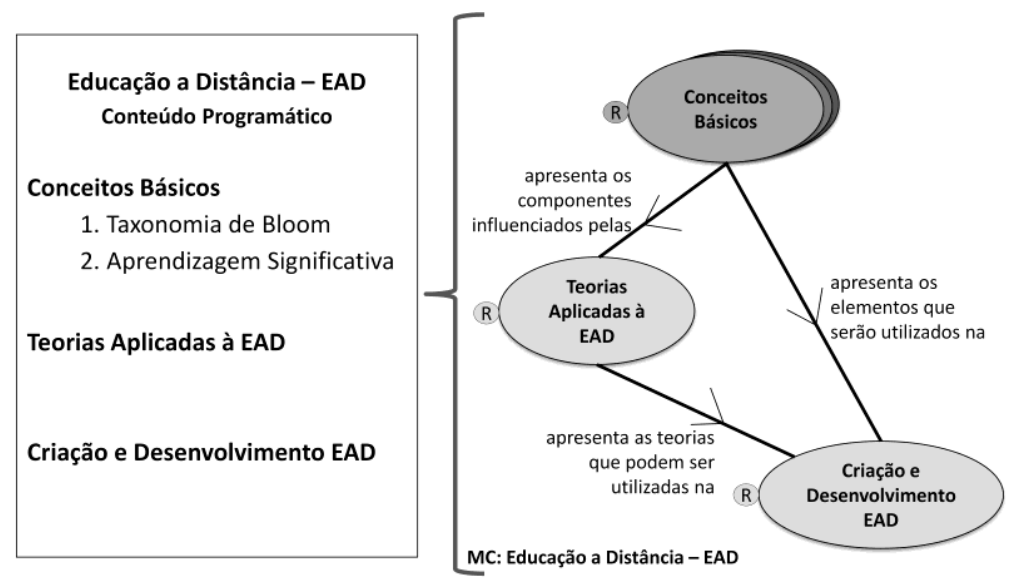

Figura 1 - Mapa de Conteúdos representando um conteúdo programático

\subsubsection{Mapas de Dependências}

Lima (2009) descreve MD como sendo uma ferramenta pedagógica formada por um conjunto de OEs apresentada de forma gráfica, onde cada OE é inter-relacionado por meio da Taxionomia de Bloom. Normalmente o MD é composto por um OE (localizado no topo do gráfico e que representa o comportamento que o professor espera que o aluno atinja para um dado conteúdo) e por um conjunto de comportamentos necessários para se atingir esse objetivo (formado por classes inferiores que contribuem para a realização do objetivo inicial).

É importante perceber que, de acordo com Lima (2009), tanto o OE quanto os comportamentos são definidos segundo as categorias que constam na Taxionomia de Bloom, podendo ter quantos subníveis forem necessários até que se atinja a classe mais baixa da taxonomia ou que o comportamento não precise de dependências. A Figura 2 ilustra o exemplo de um MD para o conteúdo “Teorias Aplicadas a EAD” apresentado na Figura 1.

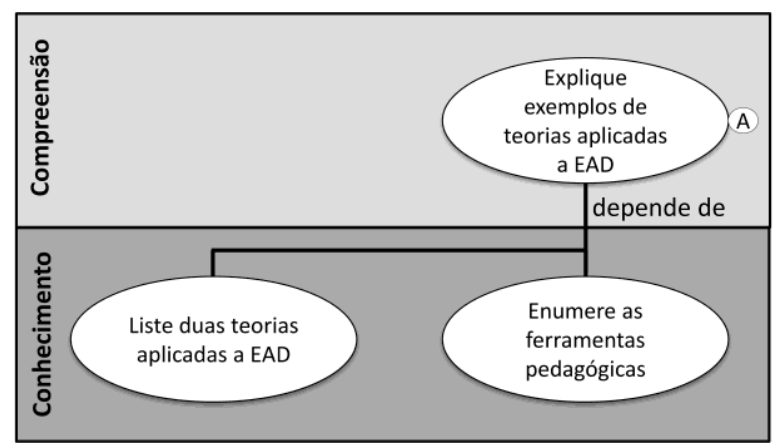

Figura 2 - Exemplo de um Mapa de Dependências

A ferramenta MD trabalha em conjunto com a ferramenta MC, possuindo também um conjunto simples de interações, onde no final do processo o gráfico com o MD terá sido gerado.

\subsection{OBA-MC}

Embora as ferramentas MC e MD, originalmente apresentadas por Lima (2009), formem um poderoso instrumento para melhoria do processo ensino-aprendizagem, elas 
possuem a limitação de funcionarem apenas no AVA Moodle. Como forma de extinguir esta restrição, Silva (2013) apresenta o OBA-MC.

OBA-MC é um modelo de Objeto de Aprendizagem (OA) em concordância com as especificações do padrão Shareable Content Object Reference Model (SCORM), que encapsula o resultado das ferramentas MC e MD em um pacote Shareable Content Object (SCO), permitindo assim, que outros sistemas (compatíveis com SCORM) possam importar e exibir corretamente os objetos desenvolvidos.

O módulo OBA-MC (adicionado ao Moodle) gera um pacote que contém um arquivo manifesto (imsmanifest.xml), um arquivo padrão de Learning Object Metadata (LOM) contendo alguns atributos relevantes que podem ser utilizados pelo sistema de busca (imslrm.xml), a estrutura do curso em eXtensible Markup Language (XML) e outros arquivos de recursos como documentos PDF, DOC, PPT, AVI, dentre outros. Para facilitar o armazenamento, transporte e recuperação, o pacote gerado é encapsulado em um arquivo ZIP.

\subsection{Mapeamento OBA-MC para XML}

Embora as ferramentas MC e MD, atualizadas por Silva (2013), gerem um pacote OBA-MC, este não pode ser utilizado diretamente pela aplicação MDC-TV. O problema é que o tamanho de um pacote OBA-MC pode ter vários Megabytes e os aparelhos de TV e set-top boxes possuem memória limitada.

A solução para diminuir o tamanho do pacote a ser utilizado pela aplicação MCD-TV, preservando todas as informações (estrutura, relacionamentos, objetivos educacionais e lista de recursos), foi encontrada por meio do mapeamento completo do pacote OBA-MC para XML.

Pela própria essência das ferramentas MC e MD, o schema utilizado para o mapeamento é uma extensão da descrição de mapas conceituais da ferramenta IHMC CmapTools, onde foram realizadas algumas inserções de novos elementos e atributos, como por exemplo: objetivo educacional e metadados do padrão Learning Object Metadata (LOM).

Para tornar o mapeamento transparente foi utilizado um Web Service que recupera objetos OBA-MC do Repositório de Objetos de Aprendizagem (ROA) e mapeia para OBA-MC XML.

\subsection{TV Digital Interativa}

Diferentemente da TV analógica, a TV Digital é vista como um agente da inclusão digital, disponibilizando aos cidadãos acesso à informação, EAD e serviços sociais apenas fazendo-se uso da própria TV (Ginga, 2012). Para isto são permitidos na TVDi quatro níveis de interatividade: (1) interatividade local; (2) com canal de retorno unidirecional; (3) com canal de retorno bidirecional; e (4) interatividade plena (Soares e Barbosa, 2009). O canal de retorno, também conhecido como canal de interatividade, é responsável por permitir que as diversas aplicações desenvolvidas possam acessar a Internet e servidores remotos existentes nas emissoras (Teleco, 2012). E que o componente responsável por facilitar o desenvolvimento de todas estas aplicações é conhecido como Ginga.

\subsubsection{Middleware Ginga}

Ginga é um middleware de especificação aberta embarcado em conversores (set-top boxes) e em televisores com tecnologia digital com interatividade, tendo duas funções 
principais: (a) tornar as aplicações independentes do sistema operacional da plataforma de hardware e (b) oferecer um melhor suporte ao desenvolvimento de aplicações interativas (DTV, 2012).

De acordo com Coulouris et al. (2007) "o termo middleware se aplica a uma camada de software que fornece uma abstração de programação, assim como o mascaramento de heterogeneidades das redes, do hardware, de sistemas operacionais e linguagens de programação”. No ambiente de TV Digital o middleware Ginga oferece, portanto, serviços padronizados à camada de aplicação, permitindo um ocultamento e abstração das camadas inferiores e seus serviços, como: compressão, transporte e modulação.

Soares (2009) explica que as aplicações desenvolvidas com a utilização do middleware Ginga podem utilizar dois ambientes distintos: o declarativo (Ginga-NCL/Lua) e o imperativo (Ginga-J/Java). As facilidades de cada um deles podem ser utilizadas pelos desenvolvedores por meio de Application Programming Interfaces (APIs) bilaterais padrões sobre um núcleo comum chamado de Ginga-CC (Common Core). A Figura 3 ilustra a arquitetura do middleware Ginga.

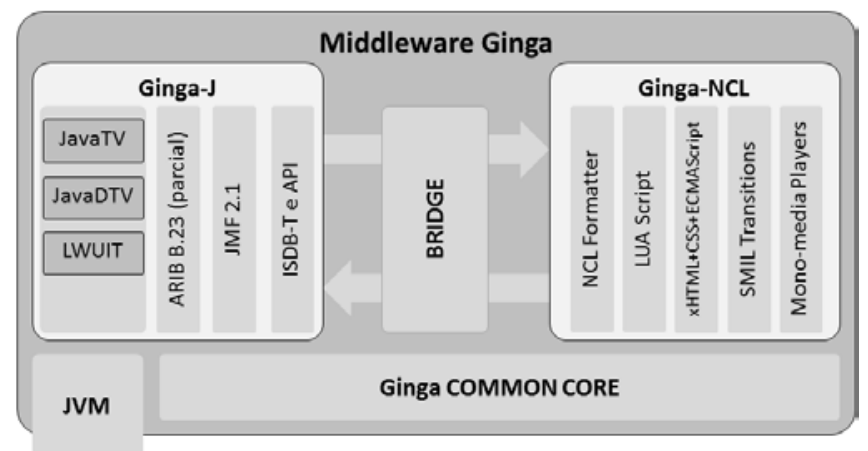

Figura 3 - Arquitetura do middleware Ginga

O ambiente utilizado neste trabalho de pesquisa é o Ginga-NCL/Lua, visto que a linguagem de script Lua é mais leve, eficiente, portável (funciona em set-top boxes e celulares com recursos para TV Digital) e livre de licença.

\subsubsection{Aplicações com Ginga}

As aplicações desenvolvidas com Ginga podem ser classificadas de acordo com o tipo de serviço, como por exemplo: (i) Serviços de Informação - EPG, notícias, previsão do tempo, informações de trânsito, entre outros; (ii) Comunicação - correio eletrônico, bate-papo, entre outros; (iii) Entretenimento - jogos, vídeo sob demanda, entre outros; (iv) Serviços Comerciais - serviços bancários, comércio eletrônico, entre outros; (v) Governo Eletrônico - portais de informações de serviços dos governos federal, estadual e municipal; (vi) T-Learning - jogos educacionais, educação básica, educação profissional, educação superior, entre outros. Este último grupo faz parte do objeto de estudo deste trabalho.

O termo T-Learning é uma especialização do conceito de E-Learning (Masie, 1999). Ele é utilizado quando se fala em aprendizagem por meio da TV Digital (Lytras et al., 2002; Zhao, 2002; Gupta, 2003). No T-Learning, não se separa TV, cultura, informação e entretenimento, sendo necessária, portanto, a criação de estratégias específicas para este ambiente, onde a presença da interatividade, de acordo com Pretto e Ferreira (2006), torna-se imprescindível, pois traz a possibilidade de alunos e professores tornarem-se autores e coautores de conhecimentos significativos. 


\subsubsection{Personalized Electronic Program Guide}

De acordo com Barbará (2010), o EPG é a aplicação para TV Digital mais comum, sendo responsável pela apresentação da programação oferecida por cada canal digital disponível. Geralmente é uma aplicação que está embarcada na TV ou set-top box, mas que também pode ser instalada pelo usuário. As informações são disponibilizadas por meio de metadados que podem conter o nome do programa, a data de exibição, o horário previsto para início e término, uma breve descrição do seu conteúdo, entre outros.

OS EPGs também podem possuir um sistema de recomendação integrado, ou seja, alguns conteúdos podem ser sugeridos ao telespectador de acordo com as suas características ou necessidades. Este tipo especial de EPG é chamado de personalized Electronic Program Guide (pEPG) (SMYTH; COTTER, 2001).

Maia et al.(2010) descreve que no SBTVD-T, a norma ABNT NBR 15603-1 (ABNT, 2007a) define tabelas que são conhecidas como Service Information (SI), entre elas a Event Information Table (EIT), que é responsável por carregar metadados dos programas que formam a programação. Os metadados são transmitidos em fluxos de transporte MPEG2-System ISO/IEC 13818-1 e seguem o formato padronizado (ABNT, 2007b). A aplicação MCD-TV utiliza os metadados (nome, tipo e descrição do programa) para fazer o relacionamento com o conteúdo do curso/disciplina selecionado pelo telespectador (aluno), com a utilização dos metadados LOM contidos no OBA-MC XML.

\subsection{Estrutura de Laboratório Utilizada}

Para o desenvolvimento da aplicação MCD-TV e realização de testes foi preciso realizar a montagem de um laboratório de TV Digital, estruturado com alguns equipamentos e softwares, entre os principais: TV Digital Interativa Full HD (para exibição da aplicação e programação televisiva); Set top-box com interatividade completa para desenvolvedor (utilizado para executar a aplicação e realização de testes); Ginga4Windows (execução/testes de aplicações NCL em ambiente Windows); NCL Composer (Ferramenta multiplataforma para criar aplicações interativas com NCL).

\section{Aplicação Interativa MCD-TV}

MCD-TV é uma aplicação Ginga-NCL/Lua desenvolvida para TVDi, que pode ser utilizada em qualquer nível de ensino e tem como base teorias pedagógicas consolidadas (Aprendizagem Significativa e Taxionomia de Bloom) e tem como objetivo trazer para o ambiente da TV uma maior significância dos conteúdos apresentados para os alunos e ampliando as possibilidades de aprendizado por meio da inclusão de programação relacionada (de qualquer emissora) aos conteúdos estudados.

Para atingir seu objetivo a aplicação faz uso de arquivo XML derivado do pacote OBA-MC (para exibição do MC e MD) e de pEPG, que permite exibir o relacionamento existente entre os conteúdos de cursos/disciplinas e a programação transmitidas pelas diversas emissoras de TV aberta. A Figura 4 apresenta o diagrama de módulos MCD-TV.

Na inicialização da aplicação MCD-TV os cursos/disciplinas transmitidos pela emissora (em formato OBA-MC XML) são lidos. Em seguida a lista de cursos/disciplinas é apresentada na tela da TV pelo módulo "Apresentador de Cursos/Disciplinas”. O telespectador, então, pode escolher qualquer um dos cursos/disciplinas listados utilizando-se apenas do controle remoto da TV. Quando um 
curso/disciplina é selecionado (tecla ENTER/OK) o módulo "Visualizador de Mapa de Conteúdos" constrói o mapa na tela de acordo com as informações contidas no OBA-MC XML. Neste momento é possível navegar entre os diversos conteúdos disponíveis, podendo-se: explorar cada um deles ou então visualizar o pEPG. Quando o telespectador estiver explorando um determinado conteúdo poderá ainda visualizar o Mapa de Dependências (caso selecione algum Objetivo Educacional).

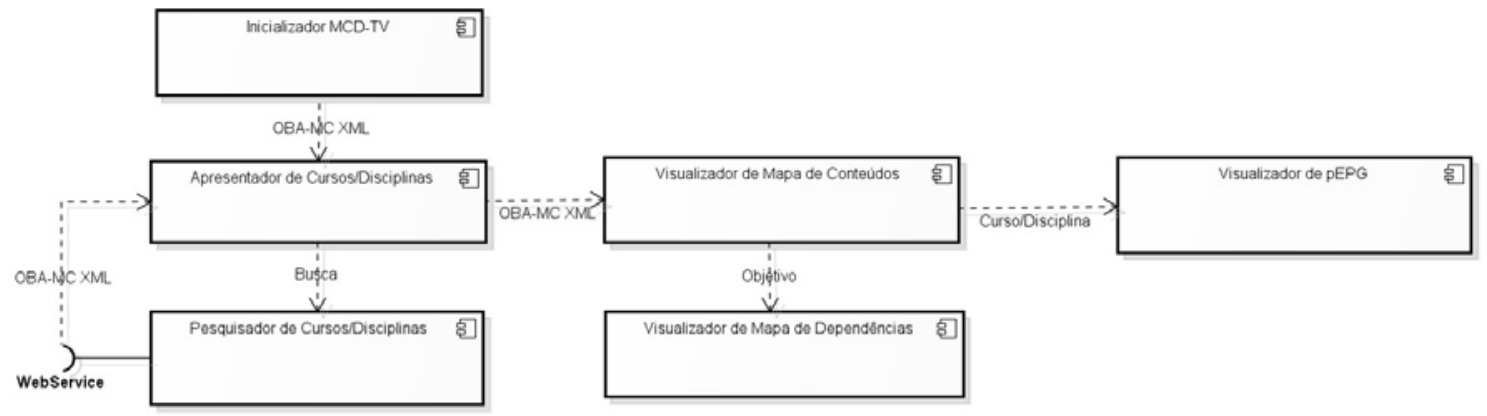

Figura 4 - Módulos componentes MCD-TV

A Figura 5 apresenta duas telas da aplicação MCD-TV. A primeira tela é exibida assim que o telespectador aciona o carregamento da aplicação. Nela é possível observar a lista de cursos/disciplinas disponíveis inicialmente; um menu de opções, acionado pelas teclas de cores do controle remoto (Vermelho - Sair, Verde - Ajuda, Amarelo - Pesquisar e Azul - Mais Informações) e a exibição da programação televisiva em tela reduzida no canto superior direito. A segunda tela é exibida após o telespectador selecionar algum curso/disciplina, apresentando o Mapa de Conteúdos. O telespectador pode navegar entre os conteúdos apresentados utilizando as setas direcionais do controle remoto da TV. Informações gerais sobre cada conteúdo também são apresentadas na tela (nome do conteúdo, tipo de conteúdo, quantidade de objetivos educacionais, total de recursos pedagógicos utilizados pelo conteúdo). Mais uma vez observa-se a existência de menu de opções, acionado pelas teclas de cores do controle remoto e a exibição da programação televisiva em tela reduzida.
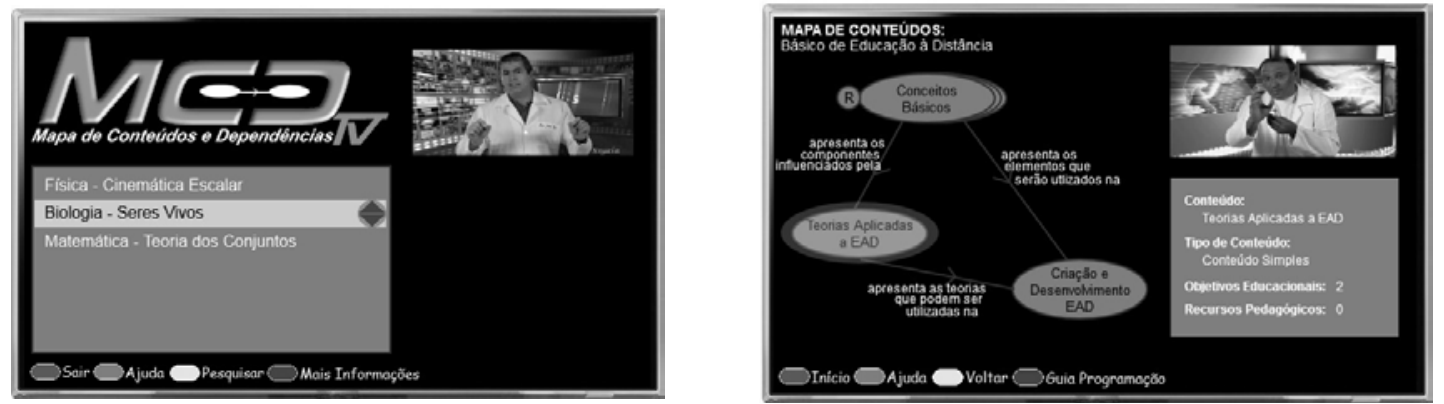

Figura 5 - Telas MCD-TV: principal e apresentação de Mapa de Conteúdos

Por questão de espaço não é possível exibir todas as telas da aplicação MCD-TV, mas todas seguem as recomendações de usabilidade para TV Digital, entre as principais: (i) posicionamento correto para exibição de textos e imagens; (ii) atenção com a diferença entre o tamanho do pixel utilizado na TV e computador; (iii) formato de tela utilizado; (iv) posicionamento e ordem de opções acionadas pelas teclas de cores; (v) estilos de fontes e tamanhos; (vi) navegação entre telas; (vii) manter padrão de telas; entre outros (CPqD, 2012; Waisman, 2006; BBC, 2006).

\subsection{Testes Realizados}

A fase de testes do protótipo MCD-TV contou basicamente com a análise de 
conformidade das recomendações de usabilidade para TVDi. Para isto foram aplicadas diversas técnicas de elicitação de interfaces, como workshops (realizados entre os membros da equipe de pesquisadores e educadores do Laboratório de Redes e Sistemas Distribuídos - LORDI/UERN), entrevistas, criação de personas e criação de protótipos de alto nível de todas as telas.

Ainda são necessários testes do protótipo fora do ambiente de laboratório para validação da interface e níveis de usabilidade, além da verificação da melhoria do processo de ensino-aprendizagem mediado pela TV em aulas reais.

\section{Considerações Finais}

A utilização da aplicação MCD-TV permite que alunos e professores tenham acesso, de forma clara e simplificada, a conteúdos mais significativos, visto que a aplicação permite que eles façam uma melhor relação entre os conteúdos apresentados na TV, os recursos pedagógicos disponibilizados e as expectativas do professor. A aplicação ainda possibilita a exibição do relacionamento existente entre os conteúdos de cursos/disciplinas ofertados com a programação televisiva oferecida pelas diversas emissoras, ampliando assim as possibilidades de aprendizado mediado pela TV. MCD-TV apresenta, portanto, um ambiente bem diferente do utilizado na TV analógica, pois traz o poder da interatividade como forma de incrementar o processo de ensino-aprendizagem.

Embora testes de interface e usabilidade tenham sido realizados, ainda se faz necessária uma investigação mais aprofundada, fora do ambiente controlado do laboratório, para verificar: nível de usabilidade, integração da arquitetura proposta aos processos de emissoras de TV, utilização da aplicação em aulas reais, entre outros.

A versão atual da aplicação também ainda possui alguns problemas: não permite a visualização, por meio da própria TV, dos diversos recursos de conteúdos disponíveis (apenas é possível a exibição de uma lista em tela dos recursos para um dado conteúdo); o professor ainda não pode alterar os mapas apresentados utilizando-se da própria aplicação MCD-TV. Espera-se resolver estes problemas nas próximas versões da aplicação. E como trabalhos futuros, pretende-se promover a criação de uma rede social de intercâmbio de conhecimento, onde professores e estudantes possam construir seus mapas e compartilhar conteúdos pelo próprio ambiente de TVDi.

\section{Agradecimentos}

Os autores agradecem a CAPES e a FAPERN pela concessão das bolsas de pesquisa e pelo apoio financeiro para realização da mesma, e em especial ao Programa de Pós-Graduação em Ciência da Computação (PPgCC) da Universidade do Estado do Rio Grande do Norte (UERN) e Universidade Federal Rural do Semi-Árido (UFERSA), por toda infraestrutura oferecida.

\section{Referências}

ASSOCIAÇÂO BRASILEIRA DE NORMAS TÉCNICAS. NBR 15603-1: televisão digital terrestre - multiplexação e serviços de informação (SI) parte 1: SI do sistema de radiodifusão. Rio de Janeiro, 2007.

NBR 15603-2: televisão digital terrestre - multiplexação e serviços de informação (SI) parte 2: estrutura de dados e definições da informação básica de SI. Rio de Janeiro, 2007. 
BARBARÁ, F. N. Aplicação Adaptativa de Guia Eletrônico utilizando o GingaNCL. Rio de Janeiro: DI/PUC-RJ, 2010. 104p. Dissertação de Mestrado.

BBC. Designing for Interactive Television v1.0. Londres, 2006. Disponível em: $<$ http://www.bbc.co.uk/guidelines/futuremedia/desed/itv/itv_design_v1_2006.pdf> Acesso em 20 maio 2012.

BLOIS, M. M. Educação a Distancia via Radio e TV Educativas: questionamentos e inquietações. Revista Em Aberto. Brasília, ano 16, n.70, abr/jun. 1996. Disponível em: $\quad<$ http://emaberto.inep.gov.br/index.php/emaberto/article/viewFile/1051/953>. Acesso em 10 mar. 2013.

BRASIL. CASA CIVIL. Decreto $\mathbf{n}^{\mathbf{0}} \mathbf{5 . 8 2 0}$, de 29 de Junho de 2006. Institui o Sistema Brasileiro de Televisão Digital - SBTVD, e dá outras providências. Disponível em: <http://www.planalto.gov.br/ccivil_03/_Ato2004-2006/2006/Decreto/D5820.htm>. Acesso em 12 out. 2012.

BRASIL. IBGE. PNAD 2011 - síntese dos indicadores. Disponível em: $<$ http://loja.ibge.gov.br/pnad-2011-sintese-dos-indicadores.html>. Acesso em mar. 2013.

COULOURIS, G.; DOLLIMORE, J.; KINDBERG, T. Distributed Systems - concepts and design. 4. Edition. [S.l.]: Bookman Companhia Ed, 2007. 784 p.

CPQD. Cartilha de Recomendações de Usabilidade para Aplicações em TVDi v1.0. Disponível em: <www.cpqd.com.br>. Acesso em 17 maio 2012.

DTV. Site Oficial da TV Digital. Disponível em: <http://www.dtv.org.br/>. Acesso em 12 jun. 2012.

GINGA. Official Site of Ginga Middleware. Disponível em: <http://http://www.ginga.org.br/>. Acesso em 12 jun. 2012.

GUPTA, M.; HUTTEMAN, K. Education with iTV. In: EUROPEAN CONFERENCE ON INTERACTIVE TELEVISION (EuroITV03), p. 111-112, 2003.

LIMA, R. W. Mapa de Conteúdos e Mapa de Dependências: ferramentas pedagógicas para uma metodologia de planejamento baseada em objetivos educacionais e sua implementação em um ambiente virtual de aprendizagem. Natal: UFRN, 2009. 106p. Tese de Doutorado.

LYTRAS, M.; LOUGOS, C.; CHOZOS, P.; POULOUDI, A. Interactive television and e-learning convergence: examining the potential of t-Learning. In: EUROPEAN CONFERENCE ON E-LEARNING, 2002.

MAIA, P. P. C.; LEITE, J.; BATISTA, T. MyPersonal-EPG: um EPG personalizável e com suporte à recomendações. In: WEBMEDIA 2010. Disponível em: <http://www.lbd.dcc.ufmg.br:8080/colecoes/webmedia/2010/07_webmi_c.pdf> Acesso: nov. 2012.

MASIE, E. et al. The computer training handbook: strategies for helping people to learn technology. Saratoga Springs: The Masie Center. 1999.

PRETTO, N. de L.; FERREIRA, S. de L. Possibilidades interativas do sistema brasileiro de televisão digital terrestre. In: $1^{\circ}$ ENCONTRO DA ULEPICCBRASIL. 2006. Niterói. 
QUIMEIA. Quimeia - preparação sem fronteiras. Disponível em: <http://www.quimeia.com.br/>. Acesso em 11 mar. 2013.

SILVA, T. R. da. OBA-MC: um modelo de objetos de aprendizagem centrado no processo de ensino-aprendizagem para o Moodle. Mossoró: UERN/UFERSA, 2013. 100p. Dissertação de Mestrado.

SMYTH, B.; COTTER, P. Personalized electronic program guides. Artificial Inteligence Magazine, 2001.

SOARES, L. F. G.; BARBOSA, S. D. J. Programando em NCL 3.0 desenvolvimento de aplicações para o middleware Ginga. Rio de Janeiro, RJ: Elsevier Editora Ltda., 2009.

TELECO. TV Digital I: introdução. Disponível em: $<$ http://www.teleco.com.br/tutoriais/tutorialinttvd1/pagina_1.asp>. Acesso em 10 jun. 2012.

WAISMAN, T. Usabilidade em Serviços Educacionais em Ambiente de TV Digital. São Paulo: Escola de Comunicação e Artes da Universidade de São Paulo, 2006. 216p. Tese de Doutorado.

ZHAO, L. Interactive Television in Distance Education: benefits and compromises. In: INTERNATIONAL SYMPOSIUM ON TECHNOLOGY AND SOCIETY (ISTAS'02), p. 255-261, 2002. 